\title{
Interactions of Bacillus mojavensis and Fusarium verticillioides with a Benzoxazolinone (BOA) and its Transformation Product, APO
}

\author{
Charles W. Bacon • Dorothy M. Hinton • \\ Anthony E. Glenn • Francisco A. Macías • David Marin
}

Received: 15 June 2007 / Revised: 25 July 2007 / Accepted: 10 August 2007 /

Published online: 25 September 2007

(C) Springer Science + Business Media, LLC 2007

\begin{abstract}
The benzoxazolinones, specifically benzoxazolin-2(3H)-one (BOA), are important transformation products of the benzoxazinones that can serve as allelochemicals providing resistance to maize from pathogenic bacteria, fungi, and insects. However, maize pathogens such as Fusarium verticillioides are capable of detoxifying the benzoxazolinones to 2-aminophenol (AP), which is converted to the less toxic $N$-(2-hydroxyphenyl) malonamic acid (HPMA) and 2-acetamidophenol (HPAA). As biocontrol strategies that utilize a species of endophytic bacterium, Bacillus mojavensis, are considered efficacious as a control of this Fusarium species, the in vitro transformation and effects of BOA on growth of this bacterium was examined relative to its interaction with strains of F. verticillioides. The results showed that a red pigment was produced and accumulated only on BOA-amended media when wild type and the progeny of genetic crosses of $F$. verticillioides are cultured in the presence of the bacterium. The pigment was identified as 2-amino-3H-phenoxazin-3-one (APO), which is a stable product. The results indicate that the bacterium interacts with the fungus preventing the usual transformation of AP to the nontoxic HPMA, resulting in the accumulation of higher amounts of APO than when the fungus is cultured alone. APO is highly toxic to $F$. verticillioides and other organisms. Thus, an enhanced biocontrol is suggested by this in vitro study.
\end{abstract}

C. W. Bacon · D. M. Hinton - A. E. Glenn

Toxicology and Mycotoxins Research Unit, Russell Research Center, USDA, ARS, Athens, Georgia 30604, USA

\section{F. A. Macías · D. Marin}

Department of Organic Chemistry, Faculty of Science, University of Cadiz, C/Republica Saharaui s/n, Apdo. 40, 11510 Puerto Real, Cadiz, Spain

C. W. Bacon $(\bowtie)$

USDA, ARS, P.O. Box 5677, 950 College Station Road, Athens, Georgia, USA

e-mail: charles.bacon@ars.usda.gov 
Keywords Allelochemicals · Aminophenoxazines - APO - Bacillus mojavensis ·

Benzoxazinoids $\cdot 2$-benzoxazolinone $\cdot 2$-amino-phenoxazin-3-one $\cdot$ BOA .

Cyclic hydroxamic acids $\cdot$ DIMBOA $\cdot$ Fusarium verticillioides $\cdot$ Maize $\cdot$ MBOA .

Microbial transformations $\cdot$ Zea mays

\section{Introduction}

Benzoxazinones and their decomposition products (benzoxazolinones and their methyl derivatives) are constitutive naturally occurring allelochemicals of wheat, corn, rye, other members of the Gramineae (Niemeyer 1988), and some non-Gramineae species (Pratt et al. 1995; Baumeler et al. 2000). These compounds serve as plant-defensive metabolites, providing protection from fungi, insects, and invertebrate pests, and they also are allelopathic. Because of their wide spectrum of biological activity, they offer promise for natural controls of pests. The main benzoxazinones produced by maize are 2,4-dihydroxy-7-mcthoxy-2H-1,4-benzoxazin3(4H)-one (DIMBOA), and its dimethoxy derivative 2,4-dihydroxy-6,7-dimethoxy-1,4benzoxazin-3(4H)-one ( $\left.\mathrm{DIM}_{2} \mathrm{BOA}\right)$, with the former occurring in greater concentrations (Whitney and Mortimore 1959; Klun and Robinson 1969; Corcuera et al. 1978; Copaja et al. 1991; Hedin et al. 1993; Frey et al. 1997; Cambier et al. 2000). These benzoxazinones are present in maize mainly as glycosides, but upon injury from insects or pathogens they are degraded to their unstable and highly toxic agluconic forms, which spontaneously decompose to the corresponding benzoxazolinones, such as MBOA (6-methoxy-2-benzoxazolinone) and BOA (benzoxazolin-2-one) (Niemeyer 1988). Thus, MBOA and BOA are found in plant debris and soil as stable degradation products of DIMBOA and DIBOA, and are considered potentially useful allelochemicals (Fomsgaard et al. 2004).

Maize begins to synthesize and store DIMBOA and $\mathrm{DIM}_{2}$-BOA as the glycosides once germination is initiated. Many insects, fungi, and bacteria are deterred or inhibited by these benzoxazinoids, resulting in increased plant resistance (Virtanen and Hietala 1955; Wahlroos and Virtanen 1958; Whitney and Mortimore 1959; Klun and Robinson 1969; Couture et al. 1971; Argandona et al. 1980; Givovich and Niemeyer 1991; Hartenstein and Sicker 1994; Koziel et al. 1996; Frey et al. 1997; Hansen 2006). The main fungal inhabitant of maize is the anamorph of Gibberella moniliformis, Fusarium verticillioides (=Gibberella fujikuroi mating population A; syn., F. moniliforme). This ascomycetous fungus is consistently found in association with maize throughout the world as a symptomless endophyte (Kommedahl and Windels 1981; Marasas et al. 1984; Bacon and Hinton 1996). The symptomless endophytic association with maize is apparently evolutionarily relevant, as it has been established that F. verticillioides and other endophytic fungi detoxify DIMBOA/MBOA and DIBOA/BOA by actively transforming them into the nontoxic components $N$-(2-hydroxy-4-methoxyphenyl) malonamic acid (HMPMA) and $N$-(2-hydroxyphenyl) malonamic acid (HPMA), respectively (Virtanen and Hietala 1955; Zikmundova et al. 2002; Glenn et al. 2003).

Fusarium verticillioides is a nonobligate pathogen; it is not host-specific and has been reported in association with many plant species in addition to maize. It is estimated that 11,665 plant species are potential hosts of $F$. verticillioides (Bacon et al. 1996), although this number may be confounded by historical confusion over current fungal and plant species delimitation and taxonomy. F. verticillioides has a long history of being associated with animal toxicosis, and only in this past decade has the etiologic agent been isolated and identified as the fumonisins (Marasas 1996). After this discovery, this class of mycotoxins was established as the cause of equine leukoencephalomalacia, porcine pulmonary edema, and experimental liver cancer in rats. The consumption of maize contaminated with this 
fungus is associated with human esophageal cancer and fetal neural tube defects (Voss et al. 1989; Wang et al. 1991; Riley et al. 1993, 1994; Waes et al. 2005). Thus, control of this cosmopolitan fungus on maize is important.

The benzoxazinoids offer protection to the plant from pathogenic attack from nondetoxifying microbes. After the very broad definition of the International Allelopathy Society (Torres et al. 1996), they can be considered allelopathic. However, the ability of fungi to detoxify these or other allelochemicals may allow for an endophytic coexistence with maize, resulting in a competitive advantage for resistant fungi (Glenn et al. 2003). Prior studies established that several species of Fusarium are tolerant to the benzoxazinoids (Glenn et al. 2001), and that this tolerance in F. verticillioides is in fact caused by the presence of at least two genetic loci, FDB1 and FDB2. Detoxification of the benzoxazolinones requires both loci, and defect at either locus causes sensitivity to the compounds (Glenn et al. 2002). A new transformation product is produced from 2aminophenol (AP). This product, 2-acetamidophenol (HPAA), was identified as a transformation compound produced by several genetic strains of $F$. verticillioides (Glenn et al. 2003). Several other fungi and bacteria are also known to detoxify this class of compounds (Friebe et al. 1996, 1998; Wieland et al. 1999; Vilich et al. 1999), although genetic mechanisms of detoxification, if any, have not been determined.

Tolerance to the benzoxazinoids by plant pathogens precludes these allelochemicals as control strategies for some pathogens. However, there are several microbial transformation products of $\mathrm{BOA}$ that are alleleopathic of which the aminophenoxazine 2-amino3H-phenoxazin-3-one (APO) (Fig. 1) is stable in soils (Macias et al. 2005). The toxicities of APO compared to other transformation products differ by several orders of magnitude higher than any other benzoxazolinone metabolites (Atwal et al. 1992; Fritz and Braun 2006; Martyniuk et al. 2006).

In a study designed to exploit the natural chemical defenses of corn in combination with the patented endophytic bacterium, Bacillus mojavensis, as a biocontrol of $F$. verticillioides, a semiochemical interaction, indicated by the formation of an intense red diffusible pigment, was observed when the two are cultured together on media amended with BOA. The endophytic bacterium has been patented for control of disease on maize, primarily control of the fungus $F$. verticillioides and mycotoxin reduction (Bacon and Hinton 1999). However, the activity of the relatively stable benzoxazolinones on this nontarget endophytic bacterium is not known, and its biocontrol activities might be compromised if it is sensitive to the benzoxazolinones and their decomposition products. As these maize allelochemicals exist in high concentrations early during maize germination (Klun and Robinson 1969) and it is at this time that the biocontrol bacterium, B. mojavensis, infects corn seedlings (Bacon and Hinton 1996), the objectives of this research were: to determine the interactive nature of $\mathrm{BOA}$ and its final transformation product, $\mathrm{APO}$, on the growth of B. mojavensis, and to

Fig. 1 Structure of 2-amino-3Hphenoxazin-3-one (APO), a degradation product of 2-benzoxazolinone (BOA)

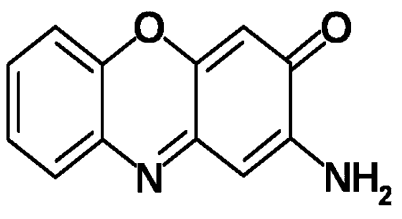

2-amino-3H-phenoxazin-3-one (APO) 
determine the in vitro antagonistic activity of the bacterium on the biocontrol target F. verticillioides cultured in the presence of BOA.

\section{Methods and Materials}

Allelochemicals Chemicals used for the transformation analysis were obtained from the following sources: BOA and 2-aminophenol from Aldrich Chemical Co., Milwaukee, WI, USA; MBOA from Lancaster Chemicals, Lancaster, Ca, USA; HPMA was purified and identified after transformation of BOA by RAC826 followed by TLC (Glenn et al. 2003). The identities of these compounds were based on prior analysis and chemical confirmation that used TLC and mass spectrometry procedures reported earlier (Glenn et al. 2003). APO was prepared and chemically characterized by previously described procedures (Macias et al. 2005).

Fungal and Bacterial Strains The strains of fungi used were those that either tested positive for detoxification of BOA or those with defective alleles at loci necessary for detoxification of BOA (Glenn et al. 2002). The F. verticillioides strains used consisted of MRC826 (genotype FDB1/FDB2), a wild type for fumonisin production and tolerance to BOA, strain AEG73-A4-1, a tolerant benzoxazolinone phenotype (genotype $F d b 1 / F d b 2$ ) derived from a cross of the wild-type $F$. verticillioides RRC826 with the female fertile AEG 3-1-6 sensitive genotype, and AEG73-A4-2 (genotype $F d b 1 / f d b 2$ ), a sensitive benzoxazolinone phenotype derived from this same cross (Glenn et al. 2002). For long-term storage, conidia were frozen at $-80^{\circ} \mathrm{C}$ in $15 \%$ glycerol. For routine culturing, spores were streaked onto potato dextrose agar (PDA; Difco Laboratories, Detroit, MI, USA) and incubated at $22^{\circ} \mathrm{C}$ in the dark. The bacterial strains used were $B$. mojavensis including the patented biocontrol strain RRC 101 (ATCC 55732), and its rifamycin-resistant mutant, RRC112 (Bacon and Hinton 2002). The bacteria were stored as silica gel cultures at $-20^{\circ} \mathrm{C}$, or $10 \%$ glycerol at $-80^{\circ}$ $\mathrm{C}$, and working cultures were maintained on nutrient agar (Difco Laboratories, Detroit, $\mathrm{MI}$, USA) at $4^{\circ} \mathrm{C}$. Inoculum of each strain was prepared from overnight shake cultures grown in nutrient broth (Difco Laboratories, Detroit, MI, USA) at $30^{\circ} \mathrm{C}$.

Bacterial-Fungal-BOA Interactions Experiments designed to determine bacterial antagonism to the fungus were assessed on nutrient agar. Antagonism experiments were performed by placing bacterial inoculum prepared from a 24-hr nutrient broth culture $\left(10^{4} \mathrm{CFU} / \mathrm{ml}\right)$ either down the center or $2 \mathrm{~cm}$ from the edge of each plate, and incubated 2$4 \mathrm{~d}$ at $30^{\circ} \mathrm{C}$. Plates were then inoculated with a fungal inoculum $\left(10^{4} \mathrm{CFU} / \mathrm{ml}\right)$ from a 5 - to 7-d-old fungal culture on either or both of the opposite edges of a Petri plate, which was incubated for $7 \mathrm{~d}$ at $25^{\circ} \mathrm{C}$. The zone of inhibition between the bacterium and fungus was measured on all test medium following earlier procedures (Bacon and Hinton 2002). Radial growth measurements were made 7 and $14 \mathrm{~d}$ after inoculation in four directions from the edge of the inoculum to the advancing margin of the colony. Additional observations were made $15 \mathrm{~d}$ after inoculations to note any differences in growth responses. Each experiment was repeated at least twice with three replications for each treatment.

Microbial Transformation in Liquid Culture Flasks, $125 \mathrm{ml}$, containing nutrient broth (50 ml/flask) amended with BOA, $1.0 \mathrm{~g} \mathrm{l}^{-1}$, were inoculated with the bacterium prepared from 24-hr nutrient broth cultures $\left(10^{6} \mathrm{cell} / \mathrm{ml}\right)$, and incubated on a shaker, $100 \mathrm{rpm}$, for $24 \mathrm{hr}$ at $25^{\circ} \mathrm{C}$. The fungal inoculum $\left(10^{4} \mathrm{cfu} / \mathrm{ml}\right)$, which was prepared from 5 - to 7 -d-old PDA cultures, was added to the BOA-amended nutrient broth immediately after the bacterial 
inoculum, placed on the shaker (150 rpm), and incubated for 5-7 d in the dark, and checked for bacterial-fungal interactions indicated by the presence of the red color of APO. Control flasks consisted of nutrient broth with and without BOA or with comparable amounts of ethanol and inoculated either with or without the bacterium or the fungus. Solvent extractions and analyses of liquid media were performed according to Yue et al. (1998). Each experiment was repeated at least twice with three replications for each treatment.

Tolerance of Bacillus mojavensis to BOA and APO Growth rate experiments were used to measure the toxicity of BOA or APO to $B$. mojavensis. Experiments were performed with an automated turbidometer, the Microbiology Bioscreen C Reader (Labsystems, Helsinki, Finland), which was conduced in 100-well sterile microplates. Each well contained $190 \mu \mathrm{l}$ of nutrient broth, with or without BOA or APO, to which was added $10 \mu$ of bacterial inoculum $\left(10^{3} \mathrm{CFU} / \mathrm{ml}\right)$ or fungal inoculum $\left(10^{6} \mathrm{CFU} / \mathrm{ml}\right)$. The cultures were incubated at $30^{\circ} \mathrm{C}$ with constant shaking, and the optical density, $\mathrm{OD}_{600}$, was measured at 30 -min intervals over the incubation period of 48-72 hr. The concentrations of BOA used were 1, 0.5 , and $0.25 \mathrm{mg} / \mathrm{ml}$. Fungal and bacterial cells from 2-wk-old BOA-amended cultures were removed by filtering through a sterile membrane $(0.2 \mathrm{~nm})$, and diluted $50 \%$ or $25 \%$ with nutrient broth. The dilutions were inoculated with either the fungus or the bacterium. Growth curves and several other plot parameters were generated and analyzed by the software package of the Bioscreen C Reader (Research Express, version 1.00). The specific rates of growth were expressed as a percent reduction over controls that contained no BOA. All experiments were performed in triplicate and were repeated independently. The reported specific rates of growth are averages from two or more experiments, and data were evaluated by analysis of variance and Student's $t$ test analyzed by the Analysis ToolPak Microsoft Excel, version 2003 (Microsoft Corp.).

Chemical Transformation of BOA in the Presence of Bacillus mojavensis The interactions of BOA metabolism in the presence of the bacterium and fungi were determined by the thin layer chromatography (TLC) procedure described earlier (Glenn et al. 2001). All strains were grown on $1.0 \mathrm{mg} / \mathrm{ml} \mathrm{BOA}$ for approximately $7 \mathrm{~d}$, and an agar plug (8 $\mathrm{mm}$ diameter) was taken from both the advancing margin of the colony and from within the colony. The agar plugs were then transferred to a TLC sheet (Whatman; silica gel, $\mathrm{UV}_{254}$, aluminum backing), which was heat activated at $50{ }^{\circ} \mathrm{C}$ for $10 \mathrm{~min}$ before application of the plugs. Plugs were placed on the sheets with the lower surface of the plugs in contact with the silica gel. The contents from plugs were allowed to absorb into the silica gel for approximately $10 \mathrm{sec}$. Three plugs from each location were placed on the same spot, with drying of the spot between each application with a heat gun, following the procedure of Glenn et al. (2001). Sheets were developed in a saturated chamber containing toluene, ethyl acetate, formic acid $(50: 40: 25, v / v)$. Developed sheets were visualized and photographed under UV light by using digital imaging (Alpha Innotech), and $R_{\mathrm{f}}$ values were compared with standards of authentic compound.

The identity of APO resulting from biotransformation of BOA by $F$. verticillioides was indicated by its bright red color (Anzai et al. 1960; Gagliardo and Chilton 1992) and by comparing TLC $R_{\mathrm{f}}$ values with standards, as well as by comparing the absorption spectra with authentic APO after eluting it with methanol from the silica gel (Glenn et al. 2003; Fomsgaard et al. 2004). Further, the confirmation of APO as the decomposition product was based on established published procedures by comparison to a standard of the authentic compound (Macias et al. 2005; Fomsgaard 2006) by using absorption spectroscopy, and gas 
chromatography-mass spectroscopy (GS-MS), described earlier (Glenn et al. 2001, 2003; Fomsgaard 2006).

\section{Results and Discussion}

The general inhibitory data and growth measurements as well as responses to the fungus by the bacterium are similar to the data described earlier (Bacon and Hinton 2002) and are pictorially shown in Fig. 2a. B. mojavensis produces a soluble inhibitory substance on nutrient agar that prevents the growth of $F$. verticillioides from making contact with the
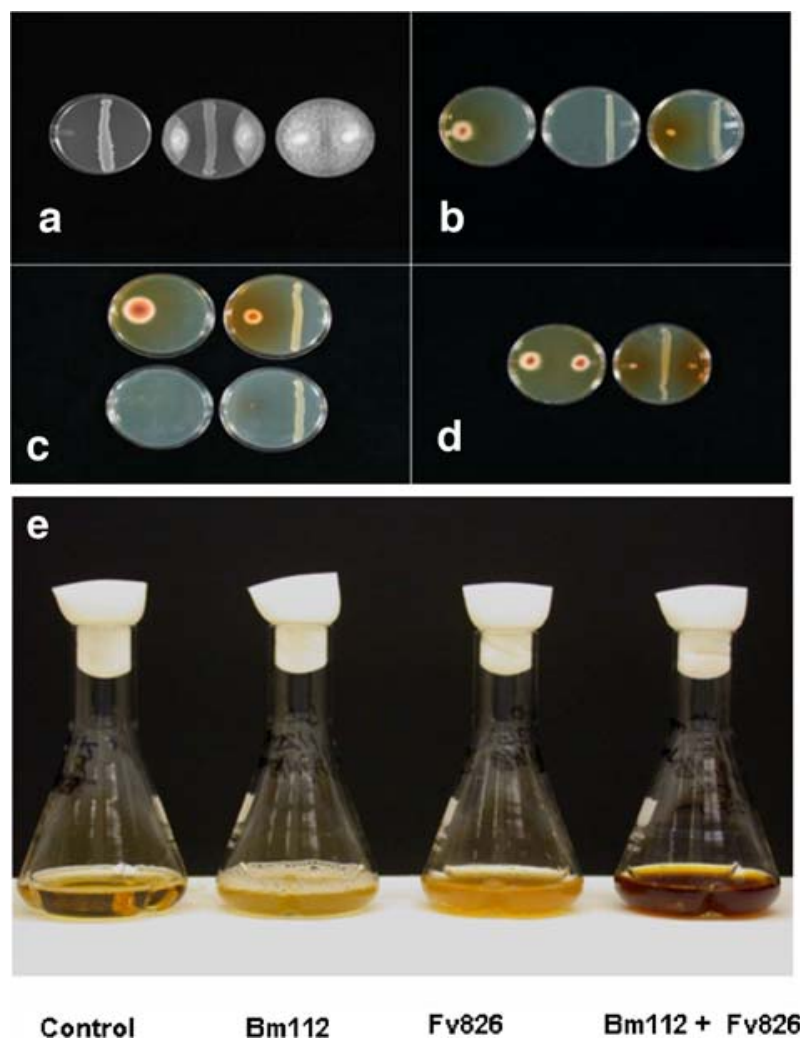

Fig. 2 Culture of Bacillus mojavensis RRC102 and Fusarium verticillioides MRC826 on nutrient agar with and without BOA. (a) Nutrient agar cultures showing zones of inhibition produced by the bacterial inhibitor after $14 \mathrm{~d}$ of culture, center plate, compared with growth of the fungus cultured alone, far right plate. (b) Culture of Fusarium verticillioides cultured with and without B. mojavensis on BOA-amended nutrient agar showing production of red pigment (APO), and on the far right is a colony of the severely inhibited fungus observed when co-cultured with the bacterium. (c). Top row, production of APO, the red pigment, by $F$. verticillioides (RRC826) with $(0.5 \mathrm{~cm})$ and without $(1.6 \mathrm{~cm})$ the bacterium B. mojavensis (RRC 112); bottom row, agar plates showing no growth with and without one of the benzoxazolinone-sensitive genetic strains of $F$. verticillioides, AEG-73-A4-2. (d) Production of APO (red pigment) in the presence of $F$. verticillioides RRC826 alone (left), and with the bacterium (right). (e) Liquid culture of B. mojavensis $($ Bm 112$)$ and $F$. verticillioides $(F v 826)$ on nutrient broth amended with BOA $\left(1.0 \mathrm{~g} \mathrm{l}^{-1}\right)$ resulting in the production of APO during single and dual culture of the organisms (Bm112 and Fv826) 
bacterial culture. This inhibitor has been partially characterized as one of the cyclic lipoprotein inhibitors characteristic of this genus of bacteria (Bacon et al. 2005). This bacterium has in vitro inhibitory activity to several known maize and other cereal pathogens (Bacon and Hinton 2002), including the ability to reduce the amount of the fumonisin mycotoxins produced by $F$. verticillioides in corn (Bacon et al. 2001). However, the interaction is complex as it was determined that $F$. verticillioides produced fusaric acid, 5-butylpicolinic acid, that is an antibiotic to B. mojavensis and other species (Bacon et al. 2006), interfering with the biocontrol potential of bacteria. The in planta accumulation of these allelochemicals, to which the Fusarium species have been shown to be tolerant (Glenn et al. 2001), is expected to complicate the use of endophytic bacteria for biocontrol work. However, the results of this study indicate that the major benzoxazolinone in maize, BOA, is not toxic to B. mojavensis, and that it is transformed in the presence of the bacterium and the fungus, resulting in a product that is further inhibitory to the fungus but not the bacterium. This is in contrast to what the fungus can do alone.

On BOA-amended medium, the inhibitory response observed on nutrient agar is intensified, and greatly restricts the growth of $F$. verticillioides compared to the inhibition observed on media without BOA (Fig. 2b). On BOA-amended medium and in the absence of the fungus, there is no production of the red pigment, even after an extended incubation period of several weeks (data not shown). During an extended incubation, the fungus degraded the BOA, producing radial growth similar to control, which was reported earlier (Glenn et al. 2001). However, on the BOA-amended medium and when both organisms are co-incubated, the red pigment is produced as early as the seventh day of incubation (Fig. 2b,c). Extraction of media, both solid and liquid, determined that this pigment formed in these cultures was APO, based on HPLC, absorption spectra, and biological activity of the compound on $F$. verticillioides. APO was isolated and chemically identified by Gagliardo and Chilton (1992) from nonsterile soil that had been supplemented with BOA. Sensitivity of $F$. verticillioides toward the inhibitory substance produced by $B$. mojavensis was enhanced greatly when cultured on the BOA-amended nutrient agar because of the production of APO. This severe inhibition was not observed on PDA amended with BOA (data not shown), as on PDA the bacterium does not produce the inhibitor. This suggests that the more restricted growth of the fungus is caused by activity of both the accumulation of APO and to the bacterial inhibitor. During an extended incubation period, the growth is still restricted when the fungus is cultured on BOA-amended media in the presence of the bacterium (Fig. 2d), and more severely restricted than when grown in the presence of the bacterium and in the absence of BOA (Fig. 2a).

An intense accumulation of APO, indicated by a large zone of deep red pigment, in the BOA-amended medium appears on media in the presence of the bacterium. The amount produced in the presence of the bacterium appears to be more, and the color is red as opposed to a yellow-red observed in culture without the bacterium (Fig. 2c,d). The inhibition observed by the bacterium (Fig. 2a) compared to that produced in the presence of BOA-amended nutrient agar is greater (Fig. 2b,d). The progeny of the two genetic crosses of $F$. verticillioides do not grow on the BOA-amended medium, and there are no accumulation patterns of the red pigments (Fig. 2c), indicating that fungal growth and activity are required for the production of transformation products from BOA. It is established that the transformation of BOA follows several steps that lead first to 2aminophenol (AP), which is a microbial transformation product (Nair et al. 1990; Gagliardo and Chilton 1992; Glenn et al. 2002; Fomsgaard et al. 2004), and subsequently microbial transformations or natural oxidative processes lead to the formation of 10 aminophenoxazinones (Fomsgaard et al. 2004). That B. mojavensis is required for the 
accumulation of APO is indicated in those cultures that showed a greater intensity of pigment accumulation over cultures that did not contain both the bacteria and fungi, and absent in nutrient broth without the addition of BOA (Fig. 2e). Another maize pathogen, Gaumannomyces graminis var. tritici, transformed BOA to APO in liquid culture (Friebe et al. 1998), and APO is now known to occur in cultures of several root endophytic fungi of tobacco, including F. sambucinum (Zikmundova et al. 2002).

The red color observed on agar media amended with BOA is also produced in nutrient broth by the bacterium-and-fungus mixed culture (Fig. 2e). Most of this color is caused by APO, which is based on its absorption spectrum of extracted media, and $R_{\mathrm{f}}$ values of the red spots on TLC plates (Fig. 3). However, there were other minor products observed on the TLC plates, suggesting the possible presence of related aminophenoxazinones. It is evident that the bacterium does not produce APO when exposed to BOA (lane 10, Fig. 3), and that APO is produced only in the presence of the fungi (lanes 4-9). Further, these data also indicate that this bacterium is not capable of transforming BOA (lane 10, Fig. 3). HPMA, a major decomposition product of BOA, was also obtained in lanes 4-9, but in the culture of the bacterium, lane 10, there was no decomposition product, and only the original BOA remained with intensity similar to the standard, lane 1 . However, the intensity of the BOA in lanes 4-9 decreased, indicating transformation by the fungus. Apparently, the tolerant cross, AEG73-A4-1, lane 9, transformed BOA similarly to the pattern identified in the wild-type strain resulting in AP, which is transformed or oxidized to APO and HPMA, and as discussed below to 2-acetaminophenol (HPAA) by strains defective at appropriate genetic loci.

APO was first described as an antifungal substance under the name of questiomycin A, a compound produced from Actinomycetes, Pseudomonas, and Waksmania aerate (Anzai et al. 1960; Gerber and Lechevalier 1964). Gagliardo and Chilton (1992) later reported that APO was more toxic to microorganisms than BOA. The toxicity spectrum for the aminophenoxazinones for those organisms tested is APO $>$ HPMA $>$ BOA (Fritz and Braun 2006). Our study, however, indicated that neither APO nor BOA was toxic to B. mojavensis (Fig. 4) at concentrations that occur naturally (Cambier et al. 2000). However, there was a significantly longer lag period at the $25-\mu \mathrm{g} / \mathrm{ml}$ concentration than obtained with the other concentrations (Fig. 4). APO was toxic to F. verticillioides when cultured on the BOAamended agar-based media used here, which suggests that the fungus was not able to

Fig. 3 TLC profiles of BOA and related metabolites obtained from agar plugs placed on silica gel plate showing the transformation products obtained from a 7-d-old culture of $F$. verticillioides MRC 826 (FV) and one of the genetic strains, AEG73-A4-1, cultured alone and in combination with $B$. mojavensis RRC112 (Bm); BOA, benzoxazolin-2-one; HPMA, $\mathrm{N}$-(2-hydroxyphenyl) malonamic acid; APO, 2-amino-3Hphenoxazin-3-one. The silica gel plates were prepared following the procedure of Glenn et al. (2001)

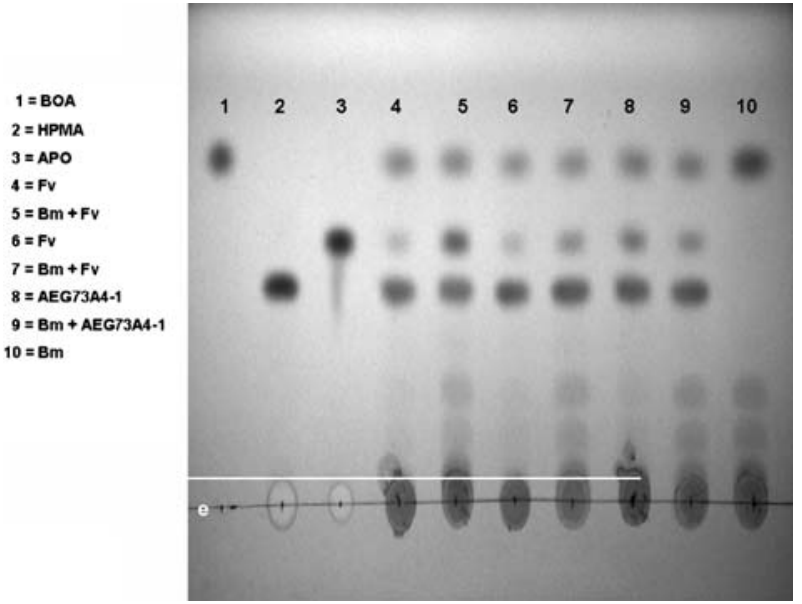


Fig. 4 Effects of APO on the specific rates of growth of Bacillus mojavensis RRC101 cultured in nutrient broth amended with different concentrations of APO $(\mu \mathrm{g} / \mathrm{ml})$. APO was solubilized in $100 \%$ ethanol (etho) and appropriate dilutions made to nutrient broth (NB); etho, ethanol control, representing the highest amount of APO added to nutrient broth during the experiment and shown for clarity as each dilution contained a comparable ethanol control; NB, nutrient broth controls

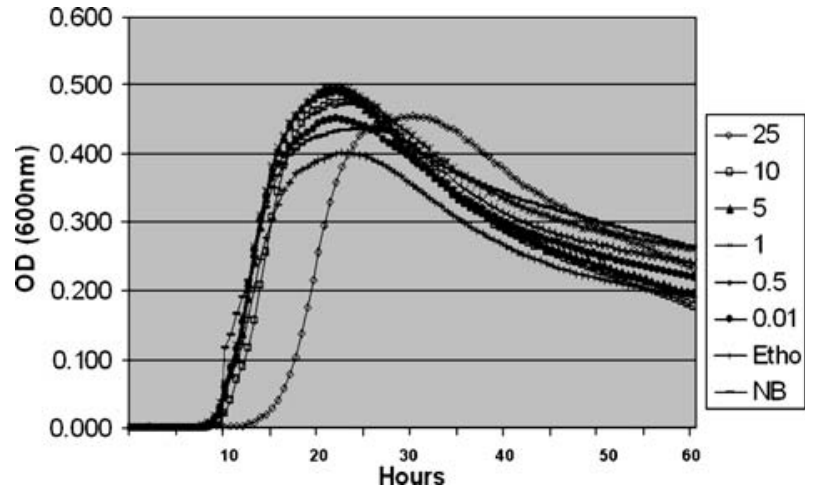

detoxify it within the 2-wk observation period. Knop et al. (2007) reported that F. verticillioides could utilize $\mathrm{APO}$ as a C-source, but indicated that its growth was reduced by $14 \%$ when cultured with $1 \mu \mathrm{M}$ of APO. This concentration was considerably less than that used here and elsewhere (Macias et al. 2005; Martyniuk et al. 2006; Krogh et al. 2006), which are concentrations reported to occur naturally (Martyniuk et al. 2006). Perhaps the growth reduction observed in F. verticillioides by Knop et al. (2007) is reflective of the small amount of APO used. However, statistical significance was not assigned to the $14 \%$ reduction observed.

We established earlier that $F$. verticillioides was capable of cleaving the oxazolone ring of BOA, producing AP that is oxidized to HPMA or HPAA, and the latter caused primarily by the inactivity of the pathway control by FDB2 (Glenn et al. 2003). It was not established that the two genetic loci have direct relationships to the accumulation pattern observed by strains of $F$. verticillioides. The two strains of $F$. verticillioides genotypes used here represented two genotypes of 24 random ascospore progeny, and each differ in the number of functional alleles at both loci, designated Fdb1 and Fdb2. Tolerant strains have both alleles at both loci, and sensitive strains are defective either at one or both loci, i.e., FDB1/ $f d b 2$ or $f d b 1 /$ FDB2. Significant amounts of HPAA are produced only by strains of the genotype Fdb1/fdb2, thus, AP is not transformed to HPAA by all strains. Further, neither the natural occurrence nor the toxicity of HPAA to other organisms has been examined. In general, however, wild-type strains of $F$. verticillioides and several Fusarium species all show tolerance to most metabolites of BOA (Yue et al. 1998; Glenn 2001; Glenn et al. 2001 ) with detoxification proceeding from BOA or MBOA resulting in the accumulation of HPMA. The primary focus of concern, therefore, is the transformation of AP to either HPMA, a substance not toxic to most naturally occurring strains of $F$. verticillioides, or to APO.

The transformation of AP to the more biologically active APO has relevance, especially at the ecological level. The ability of $F$. verticillioides to detoxify AP into several nontoxic products led to the tentative conclusion by Glenn et al. (2002) that the benzoxazolinones allelochemicals are probably not effective resistance mechanisms against this fungus. However, interactive microbial activity was not considered relevant. As established here and by others (Gagliardo and Chilton 1992; Fomsgaard et al. 2004; Fritz and Braun 2006), however, APO is an important microbial transformation product of AP, although the interactions are perhaps exceeding complexity as observed here and by others (Macias et al. 2005; Knop et al. 2007). 
Our interpretation of the results obtained in this study is that the inhibition of growth of $F$. verticillioides by $B$. mojavensis also prevents the efficient transformation of AP by $F$. verticillioides to the nontoxic HPMA, which is summarized in Fig. 5. The fungus in the presence of the bacterium seems capable of hydrolyzing BOA to AP, but then apparently is unable or at least has a reduced capacity for modifying AP to HPMA, resulting in an accumulation of AP that is either converted by the bacterium or spontaneously oxidized to APO. APO is more toxic to a broad range of microbes, including $F$. verticillioides. $B$. mojavensis is able to tolerate APO, thus an environment is created that favors the bacterium. As the effect is observed to occur some distance on culture plates from the bacterium, there is a suggestion for the production of an inhibitory diffusible substance. We speculate that if indeed an inhibitory substance is produced, it would interfere with the enzymatic function of the second genetic locus, FDB2, resulting in the accumulation of APO (Fig. 5). We do not know if this substance is the same as the fungal growth inhibitor produced in media by the bacterium that inhibits the F. verticillioides (Fig. 2a).

This is the first report to show that in the presence of a bacterium on BOA-amended medium there is an increased expression of in vitro toxicity to a fungus, which usually has the capability to detoxify BOA into decomposition products. Recently, Knop et al. (2007) demonstrated that detoxification of BOA by $F$. verticillioides was greatly reduced in the presence of the herbicide metolachlor. This information along with our data suggests that biotic and abiotic stress can influence the overall allelopathic interaction with the benzoxazolinones. As APO is the major transformation product of AP by most soil microorganisms, the role of APO and its accumulation by a fungal antagonist is highlighted here with several ecotoxicological possibilities. As B. mojavensis is an endophyte, the possible endophytic transformation of BOA into APO offers additional benefits. Further, the long-term stability of APO in soil types (Macias et al. 2005) and the suggestion that it might be the final transformation products by organisms in most soil types (Fritz and Braun 2006) are other features that should enhance the use of benzoxalinones combined with tolerant organisms such as $B$. mojavensis as effective biocontrol agents.

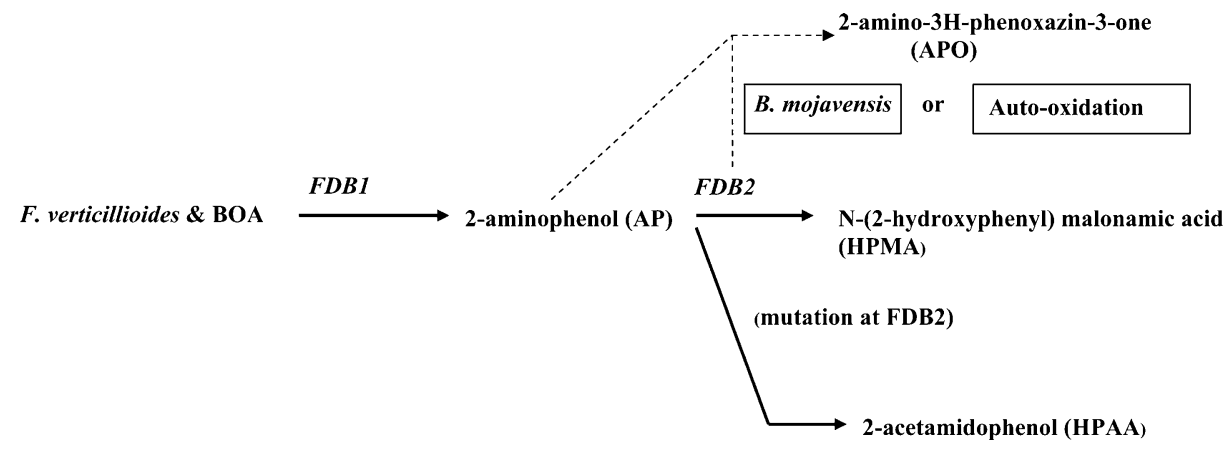

Fig. 5 Theoretical detoxification of BOA by co-cultures of Bacillus mojavensis and Fusarium verticillioides (modified from Glenn et al. 2003). F. verticillioides RRC826 cultured on BOA amended PDA transforms BOA, which involves two functional genetic loci, $F D B 1$ and $F D B 2$, leading to the production of a relatively nontoxic Fusarium transformation product, HPMA. However, a mutation at FDB2 causes the accumulation of a novel product as a result of the acetylation of AP to form HPAA. We theorize that in the presence of the wild-type loci of $F$. verticillioides, $B$. mojavensis interferes with the locus $F D B 2$, resulting in an accumulation of APO, which results either from a physical spontaneous oxidation of AP to form APO, or direct transformation by B. mojavensis to APO. Under either pathway some HPAA should also accumulate 


\section{References}

ANZAI, K., Isono, K., OKuma, K., and SuZUKI, S. 1960. The new antibiotic, Questiomycin-A and Questionmycin-B. J. Antibiot. 13:125-132.

Argandona, V. H., LuZA, J. G., Niemeyer, H. M., and Corcuera, L. J. 1980. Role of hydroxamic acids in the resistance of cereals to aphids. Phytopathology, 19:1665-1668.

Atwal, A. S., Teather, R. M., Liss, S. N., and Collins, F. W. 1992. Antimicrobial activity of 2aminophenoxazin-3-one under anaerobic conditions. Can. J. Microbiol. 38:1084-1088.

BACON, C. W., and HINTON, D. M. 1996. Symptomless endophytic colonization of maize by Fusarium moniliforme. Can. J. Bot. 74:1195-1202.

BACON, C. W., and HINTON, D. M. 1999. Use of Bacillus subtilis as an endophyte for the control of diseases caused by fungi. US Patent and Trademark Office, Patent number 5,994,117, November 30, 1999.

BACON, C. W., and HINTON, D. M. 2002. Endophytic and biological control potential of Bacillus mojavensis and related species. Biol. Control, 23:274-284.

Bacon, C. W., Porter, J. K., Norred, W. P., and Leslie, J. F. 1996. Production of fusaric acid by Fusarium species. Appl. Environ. Microbiol. 62:4039-4043.

Bacon, C. W., Yates, I. E., Hinton, D. M., and Meredith, F. 2001. Biological control of Fusarium moniliforme in maize. Environ. Health Perspect. 109:325-332.

BACON, C. W., Hinton, D. M., and SNOOK, M. E. 2005. Tentative identification of Bacillus mojavensis antifungal inhibitor. Phytopathology 95 (No. 6 Supplement), S5.

BACON, C. W., Hinton, D. M., and Hinton, A. 2006. Growth-inhibiting Effects of Concentrations of Fusaric acid on the Growth of Bacillus mojavensis and other Biocontrol Bacillus Species. J. Appl. Microbiol. 100:185-194.

Baumeler, A., Hesse, M., and Werner, C. 2000. Benzoxazinoids-cyclic hydroxamic acids, lactams and their corresponding glucosides in the genus Aphelandra (Acanthaceae). Phytochemistry, 53:213-222.

Cambier, V., Hance, T., and De Hoffmann, E. 2000. Variation of DIMBOA and related compounds content in relation to the age and plant organ in maize. Phytochemistry, 53:223-229.

COPAJA, S. V., BARRIA, B. N., and NIEMEYER, H. M. 1991. Hydroxamic acid content of perennial triticeae. Phytochemistry, 30:1531-1534.

Corcuera, L. J., Woodward, J. P., Helgeson, J. P., Kelman, A., and UpPer, C. D. 1978. 2, 4-Dihydroxy7-methoxy-2H-1, 4-benzoxazin-3(4H)-one, an inhibitor from Zea mays with differential activity against soft rotting Erwina species. Plant Physiol. 61:791-795.

Couture, R. M., RoutLey, D. G., and DunN, G. M. 1971. Role of cyclic hydroxamic acids in monogenic resistance of maize to Helminthosproium turcicum. Physiol. Plant Pathol. 1:515-521.

FOMSGAARD, I. S. 2006. Chemical ecology in wheat plant-pest interactions. How the use of modern techniques and a multidisciplinary approach can throw new light on a well-known phenomenon: Allelopathy. J. Agric. Food Chem. 54:987-990.

FomsGaARD, I. S., MoRTENSEN, A. G., and CARLSEN, S. C. K. 2004. Microbial transformation products of benzoxazolinone and benzoxazinone allelochemicals - a review. Chemosphere, 54:1025-1038.

Frey, M., Chomet, P., Glawischnig, E., Stettner, C., Grun, S., Eisenreich, W., Bacher, A., Meeley, R. B., BRIGGS, S. P., SimCOX, K., and GiERL, A. 1997. Analysis of a chemical plant defense mechanism in grasses. Science, 277:696-699.

Friebe, A., Wieler, L. H., and SchulZ, M. 1996. Tolerance of Avena sativa to the alleolochemical benzoxalinone. Degradation of BOA by root-colonizing bacteria. Angew. Bot. 70:150-154.

Friebe, A., Vilich, V., Hennig, L., Kluge, M., and SicKer, D. 1998. Detoxification of benzoxazolinone allelochemicals from wheat by Gaeumannomyces graminis var. tritici, G-graminis var. graminis, $G$ graminis var. avenae, and Fusarium culmorum. Appl. Environ. Microbiol. 64:2386-2391.

FRITZ, J. I., and BRAUN, R. 2006. Ecotoxicological effects of benzoxazinone allelochemicals and their metabolites on aquatic nontarget organisms. J. Agric. Food Chem. 54:1105-1110.

Gagliardo, R. W., and Chilton, W. S. 1992. Soil transformation of the 2(3H)-benzoxazolinone of rye into phytotoxic 2-amino-3H-phenoxazin-3-one. J. Chem. Ecol. 18:1683-1691.

Gerber, N., and LeCheVAliER, N. 1964. Phenazines and phenoxazinones from Waksmania aerata sp. nov. and Pseudomonas iodina. Biochemistry, 3:598-602.

Givovich, A., and NiemeYer, H. M. 1991. Hydroxamic acids affecting barley yellow dwarf virus transmission by the aphid Rhopalosiphum padi. Entomol. Exp. Appl. 59:79-85.

GLENN, A. E. 2001. Detoxification of corn antimicrobial compounds by the endophytic fungus Fusarium verticillioides and the significance to plant-fungus interactions. Ph.D. Dissertation, University of Georgia, Athens. 
Glenn, A. E., Hinton, D. M., Yates, I. E., and Bacon, C. W. 2001. Detoxification of corn antimicrobial compounds as the basis for isolating Fusarium verticillioides and some other Fusarium species from corn. Appl. Environ. Microbiol. 67:2973-2981.

GlenN, A. E., Gold, S. E., and BACON, C. W. 2002. Fdb1 and Fdb2, Fusarium verticillioides loci necessary for detoxification of preformed antimicrobials from corn. Mol. Plant Microb. Interact. 15:91-101.

Glenn, A. E., Meredith, F. I., Morrison, W. H. I., and BACON, C. W. 2003. Identification of intermediate and branch metabolites resulting from biotransforamtion of 2-benzoxazolinone by Fusarium verticillioides. Appl. Environ. Microbiol. 69:3165-3169.

HANSEN, L. M. 2006. Effect of 6-methoxybenzoxazolin-2-one (MBOA) on the reproduction rate of the grain aphid (Sitobion avenae F.). J. Agric. Food Chem. 54:1031-1035.

HARTENSTEIN, H., and SICKER, D. 1994. $\alpha$-hydroxylation of cyclic hydroxamic acids by peroxide oxidation: A novel approach to allelochemicals from Gramineae. Tetrahedron Lett. 35:4335-4338.

Hedin, P. A., DAVIS, F. M., and Williams, W. P. 1993. 2-Hydroxy-4, 7-dimethoxy-1, 4-benzoxazin-3-one (N-O-Me-DIMBOA), a possible toxic factor in corn to the southwestern corn borer. J. Chem. Ecol. 19:531-542.

KLun, J. A., and Robinson, J. F. 1969. Concentration of two 1, 4-benzoxazinones in dent corn at various stages of development of the plant and its relation to resistance of the host plant to European corn borer. J. Econ. Entomol. 62:214-220.

Knop, M., Pacyna, S., Voloshechuk, N., Kunt, S., Mullenborn, C., Steiner, U., Kirchmair, M. SCherer, H. W., and Schulz, M. 2007. Zea mays: Benzoxazolinone detoxification under sulfur deficiency conditions-A complex allelopathic alliance including endophytkc Fusarium verticillioides. J. Chem. Ecol. 33:225-237.

KommedAHL, T., and Windels, C. E. 1981. Root-, stalk-, and ear-infecting Fusarium species on corn in the USA, pp. 94-103, in P.E. Nelson, T.A. Toussoun, R. J. Cook (eds.). Fusarium: Diseases, Biology and Taxonomy. The Pennsylvania State University Press, University Park.

Koziel, M. G., Carozzi, N. B., Desai, N., Warren, G. W., Dawson, J., Dunder, E., Launis, K., and Evola, S. V. 1996. Transgenic maize for the control of European corn borer and other maize insect pests. Ann. NY Acad. Sci. 792:164-171.

Krogh, S. S., Mensz, S. J. M., Nielsen, S. T., Mortensen A. G., Christophersen, C., and FomsgaArd, I. S. 2006. Fate of benzoxazinone allelochemicals in soil after incorporation of wheat and rye sprouts. $J$. Agric. Food Chem. 54:1064-1074.

Macias, F. A., Oliveros-Bastidas, A., Marin, D., Castellano, D., Simonet, A. M., and Molinillo, J. M. G. 2005. Degradation studies on benzoxazinoids. Soil degradation dynamics of (2R)-2-O-B-Dglucopyranosyl-4-hydroxy-(2H)-1, 4-benzoxazin-3(4H)-one (DIMBOA-Glc) and its degradation products, phytotoxic allelochemicals from gramineae. J. Agric. Food Chem. 53:554-561.

Marasas, W. F. O. 1996. Fumonisins: History, world-wide occurrence and impact. Adv. Exp. Med. Biol. 392:1-17.

Marasas, W. F. O., Nelson, P. E., and Toussoun, T. A. 1984. Toxigenic Fusarium Species. The Pennsylvania State University Press, University Park.

Martyniuk, S., Stochmal, A., Macias, F. A., Marin, D., and OleszeK, W. 2006. Effects of some benzoxazinoids on in vitro growth of Cephalosporium gramineum and other fungi pathogenic to cereals and on Cephalosporium strip of winter wheat. J. Agric. Food Chem. 54:1036-1039.

NAIR, G., WhitenACK, C. J., and PuTnAM, A. R. 1990. 2,2'-oxo-1,1'-azobenzene, a microbially transformed allelochemical from 2, 3-benzoxazolinone: I. J. Chem. Ecol. 16:353-364.

NiEMEYER, H. M. 1988. Hydroxamic acids (4-hydro-1,4-benzozazin-3-ones), defense chemical in the Gramineae. Phytochemistry, 27:3349-3358.

Pratt, K., Kumar, P., and Chilton, W. S. 1995. Cyclic hydroxamic acids in dicotyledonous plants. Biochem. Systematics. Ecol. 23:781-785.

Riley, R. T., Norred, W. P., and Bacon, C. W. 1993. Fungal toxins in foods: Recent concerns. Annu. Rev. Nutr. 13:167-189.

Riley, R. T., Hinton, D. M., Chamberlain, W. J., Bacon, C. W., Wang, E., Merrill, A. F., JR., and Voss, K. A. 1994. Dietary fumonisin B1 induces disruption of sphingolipid metabolism in Sprague Dawley rats: A new mechanism of nephrotoxicity. J. Nutri. 124:594-603.

Torres, A., Oliva, R. M., Castellano, D., and Cross, P. I. 1996. Introduction, pp. 7, in A.Torres, R. M. Oliva, D. Castellano, and P.I. Cross (eds.). Proceedings of the First World Congress on Allelopathy-A science for the future.

Vilich, V., Löhndorf, B., Sikora, R. A., and Friebe, A. 1999. Metabolism of benzoxazolinone allelochemicals of Zea mays by Fusarium subglutinans. Mycol. Res. 103:1529-1532.

Virtanen, A. I., and HietalA, P. K. 1955. 2(3)-Benzoxazolinone, an anti-Fusarium factor in rye seedlings. Acta Chem. Scand. 9:1543-1545. 
Voss, K. A., Norred, W. P., Plattner, R. D., and Bacon, C. W. 1989. Hepatotoxicity and renal toxicity in rats of corn samples associated with field cases of equine leukoencephalomalacia. Fd. Chem. Toxic. 27:89-96.

Waes, J. G., Starr, L., Maddox, J., Aleman, F., Voss, K. A., Wilberding, J., and Riley, R.T. 2005. Maternal fumonisin exposure and risk for neural tube defects: Mechanisms in an in vivo mouse model. Teratology 73:487-497.

Wahlroos, O., and Virtanen, A. I. 1958. On the antifungal effect of benzoxazolinone and 6methoxybenzoxazolinone, respectively on Fusarium nivale. Acta Chem. Scand. 12:124-128.

Wang, E., Norred, W. P., Bacon, C. W., Riley, R. T., and Merrill, A. H., Jr. 1991. Inhibition of sphingolipid biosynthesis by fumonisins. Implications for diseases associated with Fusarium moniliforme. J. Biol. Chem. 266:14486-14490.

Whitney, N. J., and Mortimore, C. G. 1959. An antifungal substance in the corn plant and its effect on growth of two stalk-rotting fungi. Nature, 183:341.

Wieland, I., Friebe, A., Kluge, M., Sicker, D., and Schultz, M. 1999. detoxification of benzoxazolinone in higher plants, pp. 78-88, in I. Wieland, A. Friebe, M. Kluge, D. SicKer, and M. Schultz (eds.). Proceedings First World Congress on Allelopathy, Spain, Cadiz, Spain.

Yue, Q., BACON, C. W., and Richardson, M. D. 1998. Biotransformation of 2-benzoxazolinone and 6methoxy-benzoxazolinone by Fusarium moniliforme. Phytochemistry, 48:451-454.

Zikmundova, M., Brandarov, K., Bigler, L., Hesse, M., and Werner, C. 2002. Biotransformation of 2benzoxazolinone and 2-hydroxy-1, 4-benzoxazin-3-one by endophytic fungi isolated from Aplelandra tetragona. Appl. Environ. Microbiol. 68:4863-4870. 